Presented at the IEEE Nuclear Science Symposium Orlando, Florida, USA, October 25-31, 1992, and to be published in the Proceedings

\title{
Sub 60 ps Timing Resolution Using Large Area Silicon Avalance Diodes
}

\author{
Gulshan Rai \\ Lawrence Berkeley Laboratory \\ University of California, Berkeley, CA 94720 \\ and \\ A. Hauger, A. Hirsch, N. Porile, R. Scharenberg, \\ B.C. Stringfellow, and M. Tincknell \\ Purdue University \\ West Lafayette, IN 47907-1396
}

October 1992 


\title{
SUB 60 ps TIMING RESOLUTION USING LARGE AREA SILICON AVALANCHE DIODES
}

\author{
A. Hauger, A. Hirsch, N. Porile, R. Scharenberg, B. C. Stringfellow, and M. Tincknell \\ Purdue University, Physics Dept. \\ West Lafayette, IN 47907-1396 \\ G. Rai \\ Lawrence Berkeley Laboratory \\ University of California, Berkeley, CA 94720
}

\section{Introduction}

The new generation of planned experiments in high energy and nuclear physics requires high pixel density time-of-flight detection systems which mark the occurrence of an event with sub- 100 ps accuracy. Conventional fast phototubes and scintillators do not easily lend themselves to large scale mechanical and electrical integration and generally prove to be very expensive.

As an alternative technology, we report on the development and optimization of Silicon Avalanche Diodes (AVDs). The project is in part strongly motivated by the STAR experiment planned for the Relativistic Heavy Ion Collider (RHIC) facility at BNL. The time-of-flight component of STAR is expected to operate in a $0.5 \mathrm{~T}$ magnetic field and is highly segmented.

Two applications are being considered. The first concept relies on the large number of electron-nole pairs $(\sim 100$ pairs $/ \mu \mathrm{m})$ created along the path of a particle passing through the $\mathrm{AVD}$ and on the various collection times which are inherently short. Using modern epitaxial techniques it is possible to produce a 4 " silicon wafer with approximately $50 \mathrm{AVDs}$ arranged in clusters of four. The ancillary readout electronics for each cluster would be located directly behind the first wafer. The second idea utilizes the AVD as the active element in a novel kind of hybrid AVD photomultiplier tube. The concept of making hybrid tubes with ordinary silicon diodes is not new 1 . There is currently a widespread interest in proximity-focused hybrid PMTs for use in single photon counting, in spectroscopy, and in calorimetry ${ }^{2}$. However, our AVD hybrid scheme emphasizes high speed timing. Initially an incoming photon is converted on a photocathode into an electron, which in turn is accelerated over a small vacuum gap and imbedded in the depletion layer of the AVD. Since the penetration is a few $\mu \mathrm{m}$ deep, only a local "ball" of ionization is deposited just beneath the surface of the AVD. The ionization electrons are swept into the avalanche multiplication region without suffering serious diffusion. In principle therefore, the AVD not only provides favorable gain but additionally a fast timing signal with low transit time jitter.

In this paper we describe the progress made in a step-by-step program to develop and evaluate prototype devices. As our first goal we have examined commercially available low $\mathbf{k}(\sim$ 0.02 ) value AVDs which could, in the short term, be adapted and tested for timing characteristics and verify a proof of principle.

\section{Avalanche Diode Description}

Large area $\left(5 \times 5 \mathrm{~mm}^{2}\right.$ sensitive area, 120 $\mu \mathrm{m}$ thick, $\mathrm{C}=20 \mathrm{pF}$ ) photo avalanche diodes of the "reach through" geometry were purchased from EG\&G Optoelectronics Division, Montreal, Canada ${ }^{3}$ and assembled with the following modifications:

a. a thin metallic layer was added to the p+ side of the AVD to decrease the spreading resistance,

b. each AVD was mounted in a sealed $3.8 \times 13 \times 33 \mathrm{~mm}^{3}$ metal enclosure together with a low noise, $100 \mathrm{MHz}$ bandwidth, transimpedance preamplifier,

c. a glass window, normally set in front of the AVD to transmit optical light to the diode, was replaced by an opaque Beryllium window 1 mil thick to allow the passage of electrons with minimal multiple scattering, 
d. two capacitors were added on opposite edges of the diode to buffer the high voltage; this arrangement minimized signal transit time across the diode surface.

\section{Performance and Results}

The tests consisted of exposing two AVDs in coincidence to minimum ionizing particles and recorded the time difference for several thousand events. The minimum ionizing particles were beta electrons from a $106 \mathrm{R} u$ source which has an end point energy of 3.5 $\mathrm{MeV}$. These particles were collimated, sent through the AVDs and stopped in a plastic scintillator coupled to a PMT (Figure 1). A pulse height cut on the scintillator signal selected the high energy tail of the beta spectrum and a triple coincidence was required among the AVDs and the scintillator to trigger an event. The electronics consisted of commercially available NIM modules. With this system, the electronic time dispersion per channel was determined to be 5.4 ps.

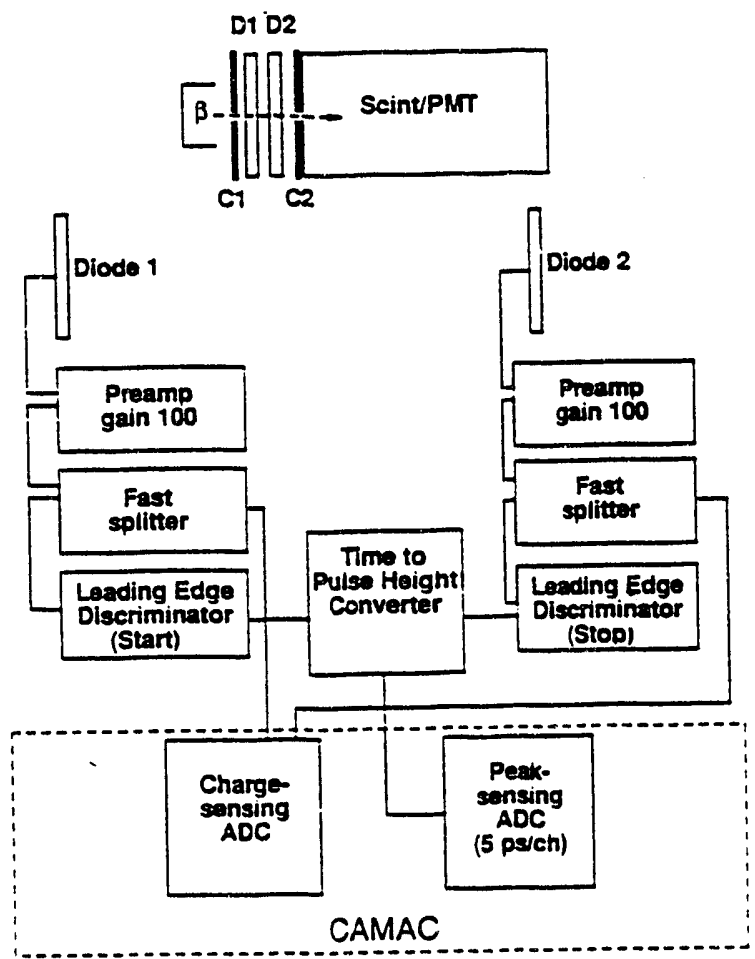

Figure 1. Schematic Diagram of the Experimental Setup and the Electronics.

The pulse shape of the beta signal, signalto-noise ratio and the coincidence time spectrum of two AVDs have been measured. The observed results are generally consistent with theoretical expectations. Other measurements include the variation of pulse height as a function of the applied bias voltage and the determination of breakdown voltage versus temperature in the range -15 to $45{ }^{\circ} \mathrm{C}$ (Figure 2). The time resolution was found not to change dramatically with temperature. This lack of sensitivity is not yet fully understood, but the result is encouraging since it implies that AVD TOF detectors may not require expensive or difficult stabilization of either the bias or ambient temperature.

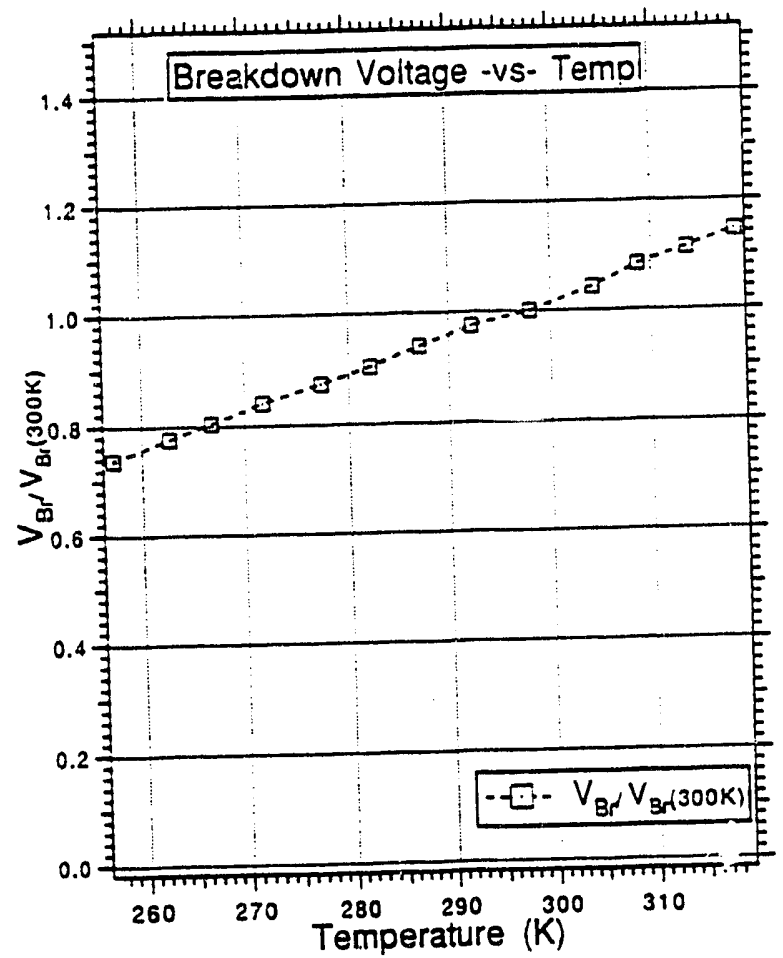

Figure 2: Breakdown Voltage vs. Temperature.

A single diode resolution of 60 ps was obtained using fast leading edge discriminators (Figure 3). This is our major result. The AVD current gain was also measured as function of bias voltage and is reproduced in Figure 4. These are preliminary. results and the absolute gain might be a little different because of systematic uncertainties. However, our gain measurements are smaller than values obtained using single photons at the same bias voltage. In fact, an order of magnitude disparity exists near the breakdown voltage. The multiplication process apparently depends on the primary current density injected into the avalanche region. This effect, although not entirely understood, might be explained in part by space charge in the 
avalanche region. Further simulation studies are in progress.

Simple calculations lead us to believe that better performance is still possible with further optimization of the AVD parameters, and improvements made to the preamplifier electronics. Timing resolution is essentially determined by the rise-time of the AVD output pulse and the signal-to-noise ratio at the leading edge discriminator threshold. Avalanche diodes with super low $\mathbf{k}$ values are expected to be less noisy. Therefore, we plan to investigate the large area $200 \mathrm{~mm}^{2}$ ( $16 \mathrm{~mm}$ diameter) diodes produced by Advanced Photonix Inc., which offer a maximum small signal gain of 1000 and a $\mathbf{k}$ value of 0.001 . These diodes are of the deep diffusion beveled edge type. Radiation damage is of particular concern to us because of its intended application in an accelerator environment. We shall study the AVDs after exposure to different levels of radiation.

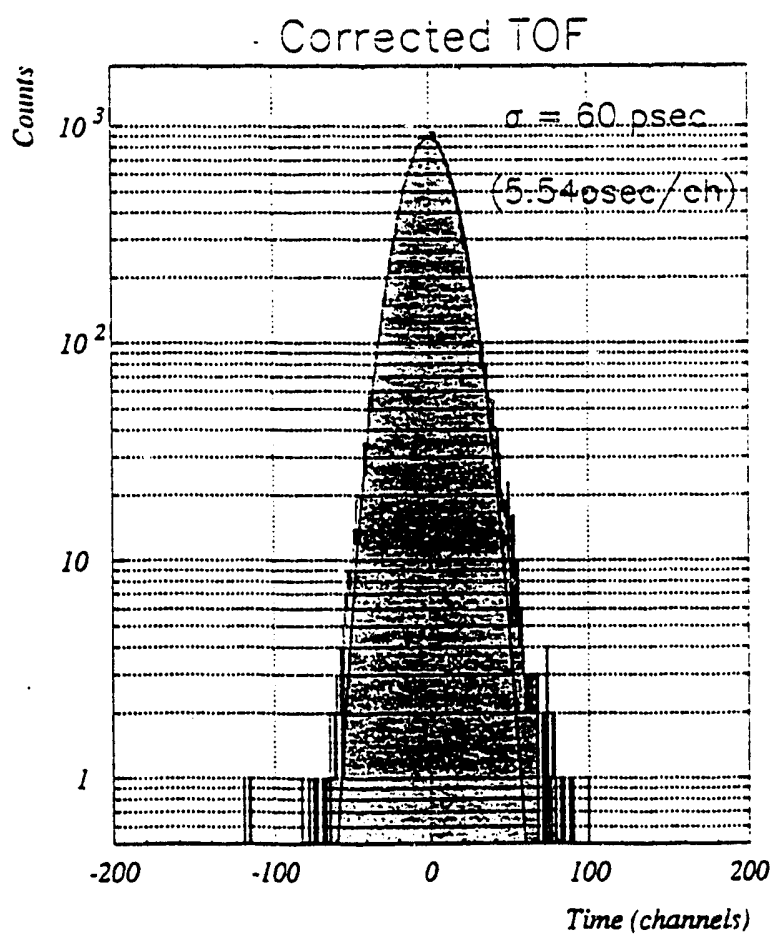

Figure 3. Time-of-flight Coincidence Spectrum after Correcting for Slewing Effects.

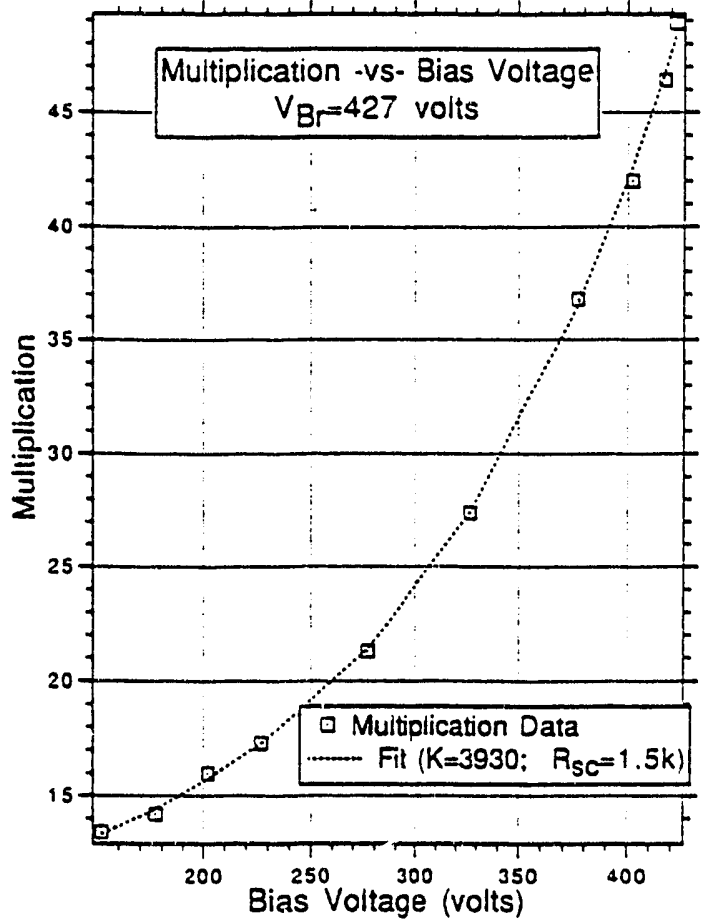

Figure 4. Multiplication vs. Bias Voltage.

${ }^{1}$ R. DeSalvo, W. Hao, K. You, Y. Wang, C. Xu, submitted to NIM, 1991.

2P. Cushman, M. Iosue, B. Johnson, $M$. Madden, R. Rusack, M. Szawlowski, to be published, 1992.

3G.A. Petrillo \& R.J. McIntyre, IEEE Trans.

Nucl. Sci., 31, 417, (1984). 

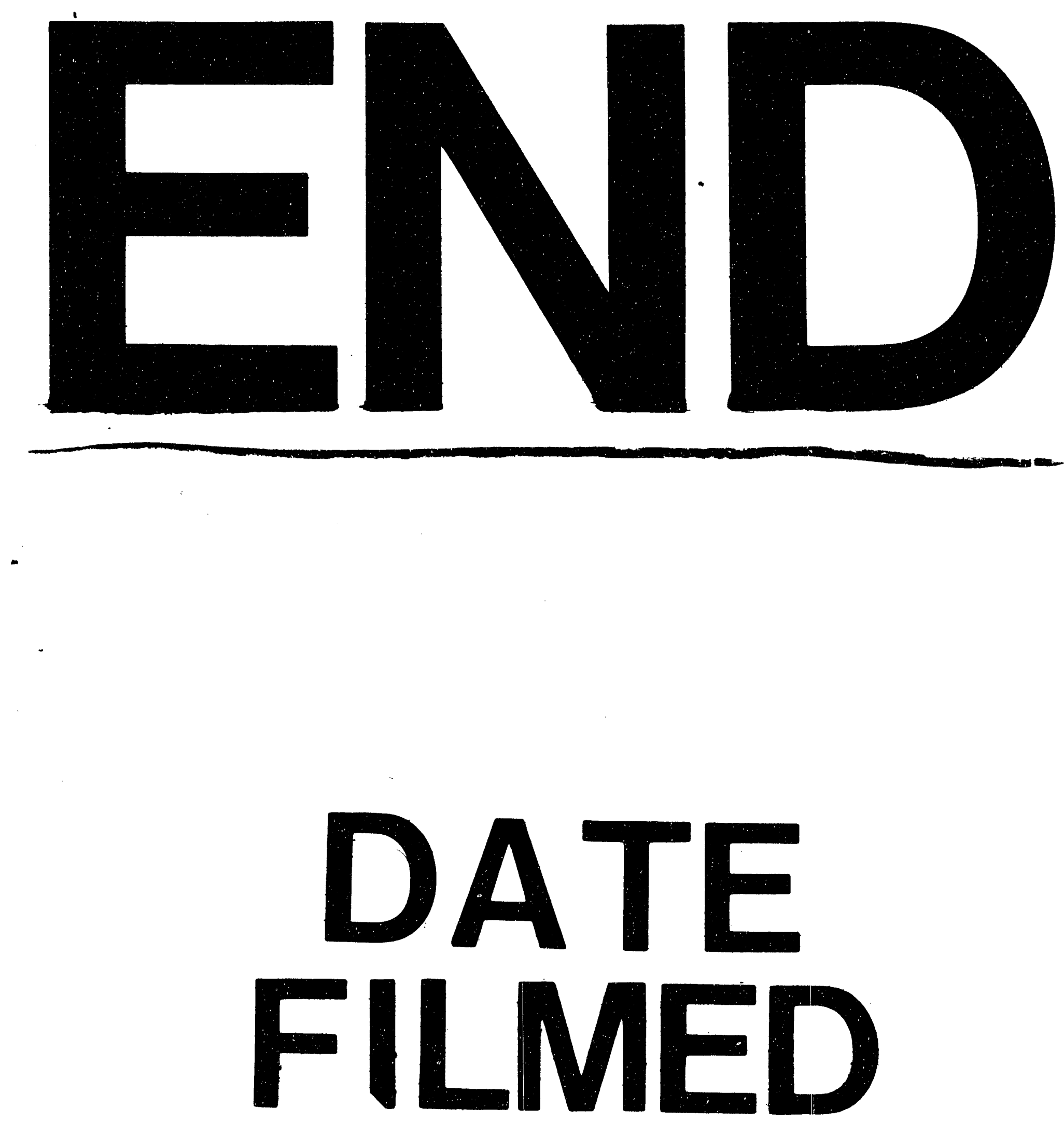

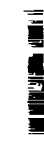

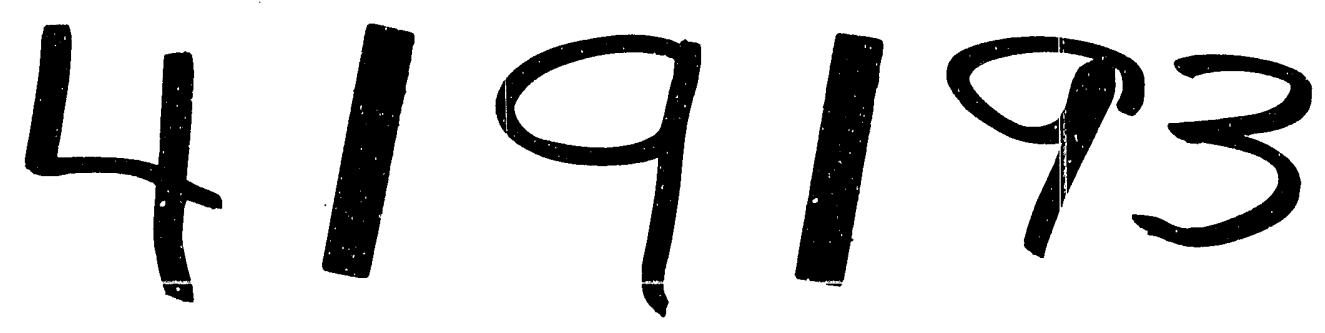


